\title{
ORIGINAL
}

\section{Predictive impact of adiponectin for detecting dysglycemia in non-fasting Japanese employees with metabolic risk factors}

\author{
Mitsuyoshi Takahara $^{1)}$, Naoto Katakami ${ }^{1), 2)}{ }^{2}$, Hideaki Kaneto ${ }^{1)}$, Midori Noguchi ${ }^{3)}$ and Iichiro Shimomura ${ }^{1)}$ \\ 1) Department of Metabolic Medicine, Osaka University Graduate School of Medicine, Osaka, Japan \\ 2) Department of Metabolism and Atherosclerosis, Osaka University Graduate School of Medicine, Osaka, Japan \\ ${ }^{3)}$ Health Support Promotion Section, Environment and Civic Affairs Bureau, Amagasaki City Office, Hyogo, Japan
}

\begin{abstract}
The screening of dysglycemia in the non-fasting state is a challenging issue in healthcare practice. We investigated whether the additional measurement of circulating adiponectin levels could improve screening performance. We used a database of 1190 Japanese employees with metabolic risk factors, who underwent a 75-g oral glucose tolerance test (OGTT), following non-fasting health check-ups. Dysglycemia was defined as fasting glucose levels $\geq 6.1 \mathrm{mmol} / \mathrm{L}$ or 2-hr glucose levels $\geq 7.8 \mathrm{mmol} / \mathrm{L}$ during the OGTT. Logistic regression analysis revealed that decreased adiponectin levels were associated with dysglycemia, independently of postprandial glucose (PG) and hemoglobin A1c (HbA1c) levels, as well as other health check-up data $(p<0.01)$. The trivariate model with PG, HbAlc, and adiponectin levels $(\mathrm{PG}+\mathrm{A} 1 \mathrm{c}+\mathrm{ADN}$ model) had a larger area under the receiver operating characteristic curves (AUC) than the bivariate model with only PG and $\mathrm{HbA} 1 \mathrm{c}$ levels $(0.746$ vs. $0.729 ; p=0.01)$. However, the AUC of the multivariate model with PG, HbA1c, and other health check-up data, but not adiponectin levels ( $\mathrm{PG}+\mathrm{A} 1 \mathrm{c}+$ Other model) was 0.815 , much larger than that of the $\mathrm{PG}+\mathrm{A} 1 \mathrm{c}+\mathrm{ADN}$ model $(p<0.01)$. The addition of adiponectin levels to the $\mathrm{PG}+\mathrm{A} 1 \mathrm{c}+$ Other model had a significantly larger AUC than the A1c $+\mathrm{PG}+$ Other model only in the subgroup without abdominal obesity $(p=0.01)$, but not in the overall population $(p=0.06)$ or in the subgroup with abdominal obesity $(p=0.62)$. In conclusion, circulating adiponectin levels were independently associated with dysglycemia in non-fasting Japanese employees with metabolic risk factors, but they improved the screening capacity only in those without abdominal obesity.
\end{abstract}

Key words: Dysglycemia, Screening performance, Non-fasting state, Adiponectin

ALTHOUGH workplaces are one of the best places to screen people for their risk of lifestyle-related diseases, including glucose intolerance and diabetes mellitus [1], some employees have difficulty in undergoing health check-ups in the fasting state and prefer non-fasting examinations. In these subjects, the risk of dysglycemia is often assessed by postprandial plasma glucose levels as well as hemoglobin A1c levels. However, the screening performance of postprandial glucose levels is inferior compared to fasting glucose levels [2], and screening of dysglycemia in the non-fasting state is still one of the challenging issues in healthcare practice.

Recent studies have revealed that decreased circulating adiponectin levels are associated with glucose

Submitted Apr. 1, 2013; Accepted Apr. 30, 2013 as EJ13-0139 Released online in J-STAGE as advance publication May 17, 2013

Correspondence to: Mitsuyoshi Takahara, M.D., Ph.D., Department of Metabolic Medicine, Osaka University Graduate School of Medicine, 2-2 Yamadaoka, Suita, Osaka 565-0871, Japan.

E-mail: takahara@endmet.med.osaka-u.ac.jp intolerance and diabetes mellitus [3-8]. However, it remains unclear whether its measurement would be useful for the screening of dysglycemia. The aim of the current study was to investigate whether the additional measurement of circulating adiponectin levels could improve the screening performance for dysglycemia in Japanese employees attending health checkups in the non-fasting state.

\section{Materials and Methods}

\section{Study population and definitions}

We used a cross-sectional database of the Amagasaki Visceral Fat Study, registered as UMIN000002391. The study was approved by the human ethics committee of Osaka University and written informed consent was obtained from every participant. The study subjects were a total of 1190 Japanese employees of the city office of Amagasaki, Hyogo, who had never been diagnosed or treated with diabetes mellitus. All the subjects 
underwent a 75-g oral glucose tolerance test (OGTT), following a non-fasting health check-up. The subjects had one or more of the following metabolic risk factors: (1) postprandial glucose levels $\geq 7.8 \mathrm{mmol} / \mathrm{L}$ (140 mg/ dL), (2) hemoglobin A1c levels $\geq 5.6 \%$, (3) elevated blood pressure (systolic blood pressure $\geq 130 \mathrm{mmHg}$ or diastolic blood pressure $\geq 85 \mathrm{mmHg}$, or taking medications of hypertension), (4) abnormal lipid metabolism (high-density-lipoprotein (HDL) cholesterol levels < $1.0 \mathrm{mmol} / \mathrm{L}$ ( $40 \mathrm{mg} / \mathrm{dL}$ ), postprandial triglycerides levels $\geq 2.3 \mathrm{mmol} / \mathrm{L}$ ( $200 \mathrm{mg} / \mathrm{dL})$, or taking medications of dyslipidemia), (5) body mass index (BMI) $\geq 25 \mathrm{~kg} / \mathrm{m}^{2}$, (6) abdominal obesity (waist circumference $\geq 85 \mathrm{~cm}$ in males and $\geq 90 \mathrm{~cm}$ in females), and/or (7) serum alanine aminotransferase levels $\geq 31 \mathrm{U} / \mathrm{L}$ [9-15]. We included these subjects, simply because they were considered to have a high risk for dysglycemia, and to be a high priority of the screening. Indeed, OGTT is recommended in those with postprandial glucose levels $\geq 7.8 \mathrm{mmol} / \mathrm{L}$ or hemoglobin A1c levels $\geq 5.6 \%$ [9]. Furthermore, it is known that metabolic syndrome and obesity are associated with insulin resistance and diabetes mellitus [14-17]. It is also known that individual components of metabolic syndrome are associated with insulin resistance [17-19]. We therefore adopted these components as inclusion criteria in the current study. We used $2.3 \mathrm{mmol} / \mathrm{L}(200 \mathrm{mg} / \mathrm{dL})$ as a cutoff point of postprandial triglycerides levels [10], providing a high specificity for hypertriglyceridemia defined by fasting triglycerides levels $\geq 1.7 \mathrm{mmol} / \mathrm{L}(150 \mathrm{mg} / \mathrm{dL})$ [20]. We also adopted elevated alanine aminotransferase levels as an inclusion criterion, because alanine aminotransferase is known to be a marker of non-alcoholic fatty liver disease, which is associated with insulin resistance and diabetes mellitus [11-13]. Note that these data were obtained in the preceding non-fasting health check-up (see below). Subjects with hepatic disease, renal disease, and/or malignant neoplasms were excluded for the current study.

In the preceding health check-ups, anthropometric measurements, sphygmomanometry, and laboratory examinations, as well as checking of medical history, were performed. Laboratory examinations included plasma glucose, hemoglobin A1c, serum total cholesterol, HDL cholesterol, triglycerides, transaminase, and adiponectin levels. Serum concentrations of adiponectin were measured by a latex particle-enhanced turbidimetric assay (Otsuka Pharmaceutical and Mitsubishi Chemical Medience, Tokyo, Japan) [21]. Hemoglobin
A1c levels were measured by high-performance liquid chromatography (HPLC) and were converted to a National Glycohemoglobin Standardization Program equivalent value with the conversion equation reported by the Japan Diabetes Society [22].

The outcome measure was dysglycemia, determined by the subsequent 75-g OGTT as either fasting plasma glucose levels $\geq 6.1 \mathrm{mmol} / \mathrm{L}(110 \mathrm{mg} / \mathrm{dL})$ or 2 -hour plasma glucose levels $\geq 7.8 \mathrm{mmol} / \mathrm{L}(140 \mathrm{mg} / \mathrm{dL})$ (i.e., not "normal type") [9]. We investigated whether circulating adiponectin levels would have a significant association with, and have a significant predictive performance for the outcome.

\section{Statistical analysis}

Logistic regression models were used to develop predictive tools for detecting undiagnosed dysglycemia. The dependent variable was the presence of OGTT-detected dysglycemia and the explanatory variables were the laboratory and non-laboratory data obtained from the preceding health check-ups. We first developed a bivariate model in which explanatory variables were plasma glucose and hemoglobin A1c levels (PG+A1c model). We subsequently developed a trivariate model which also included adiponectin levels (PG+A1c+ADN model). We also developed a multivariate stepwise model in which other health check-up data instead of adiponectin levels were added to plasma glucose and hemoglobin A1c levels $(\mathrm{PG}+\mathrm{A} 1 \mathrm{c}+$ Other model). The candidates for the explanatory variables other than plasma glucose and hemoglobin A1c levels were: sex, age, BMI, systolic blood pressure, serum total cholesterol, HDL cholesterol, triglycerides, alanine aminotransferase, and presence of hypertension and dyslipidemia under treatment. We finally developed a full model in which both adiponectin levels were added to the $\mathrm{PG}+\mathrm{A} 1 \mathrm{c}+$ Other model (Full model). The predictive capability of these developed models for dysglycemia was evaluated using receiver operating characteristic (ROC) curves, and the area under the curves (AUC) was calculated. We also investigated the predictive capability of the subgroups with and without abdominal obesity.

Data are given as means and standard deviations (SD) for continuous variables or as percentages for dichotomous variables, if not mentioned otherwise. Serum adiponectin levels, as well as serum triglycerides and alanine aminotransferase levels, had a rightskewed distribution and therefore was log-trans- 
Table 1 Characteristics of the study population

\begin{tabular}{|c|c|c|c|c|}
\hline & $\begin{array}{l}\text { Overall population } \\
\quad(n=1190)\end{array}$ & $\begin{array}{c}\text { Subjects without } \\
\text { dysglycemia }(n=894)\end{array}$ & $\begin{array}{c}\text { Subjects with } \\
\text { dysglycemia }(n=296)\end{array}$ & $p$ value \\
\hline Males & $1015(85 \%)$ & $759(85 \%)$ & $256(86 \%)$ & 0.57 \\
\hline Age (years) & $50 \pm 9$ & $48 \pm 9$ & $54 \pm 7$ & $<0.01$ \\
\hline Body mass index $\left(\mathrm{kg} / \mathrm{m}^{2}\right)$ & $25.1 \pm 3.2$ & $24.9 \pm 2.9$ & $25.7 \pm 3.7$ & $<0.01$ \\
\hline Systolic blood pressure (mmHg) & $130 \pm 18$ & $128 \pm 17$ & $136 \pm 19$ & $<0.01$ \\
\hline Plasma glucose $(\mathrm{mmol} / \mathrm{L})$ & $5.8 \pm 1.1$ & $5.6 \pm 1.0$ & $6.3 \pm 1.3$ & $<0.01$ \\
\hline Hemoglobin A1c (\%) & $5.6 \pm 0.4$ & $5.5 \pm 0.4$ & $5.8 \pm 0.4$ & $<0.01$ \\
\hline Total cholesterol (mmol/L) & $5.4 \pm 0.9$ & $5.4 \pm 0.9$ & $5.5 \pm 1.0$ & 0.13 \\
\hline HDL cholesterol (mmol/L) & $1.4 \pm 0.4$ & $1.4 \pm 0.4$ & $1.4 \pm 0.4$ & 0.27 \\
\hline Triglycerides $(\mathrm{mmol} / \mathrm{L})$ & $1.8(1.2-2.7)$ & $1.7(1.1-2.6)$ & $2.1(1.4-2.8)$ & \\
\hline Log $($ Triglycerides $(\mathrm{mmol} / \mathrm{L}))$ & $0.25 \pm 0.26$ & $0.23 \pm 0.26$ & $0.29 \pm 0.24$ & $<0.01$ \\
\hline Alanine aminotransferase (IU/L) & $21(16-34)$ & $21(15-32)$ & $26(19-41)$ & \\
\hline Log (Alanine aminotransferase (IU/L)) & $1.37 \pm 0.23$ & $1.35 \pm 0.23$ & $1.44 \pm 0.25$ & $<0.01$ \\
\hline Adiponectin $(\mu \mathrm{g} / \mathrm{mL})$ & $6.3(4.7-8.3)$ & $6.4(4.8-8.6)$ & $6.0(4.4-7.6)$ & \\
\hline $\log ($ Adiponectin $(\mu \mathrm{g} / \mathrm{mL}))$ & $0.80 \pm 0.20$ & $0.81 \pm 0.20$ & $0.76 \pm 0.19$ & $<0.01$ \\
\hline Hypertension under treatment & $169(14 \%)$ & $92(10 \%)$ & $77(26 \%)$ & $<0.01$ \\
\hline Dyslipidemia under treatment & $52(4 \%)$ & $31(3 \%)$ & $21(7 \%)$ & 0.01 \\
\hline
\end{tabular}

Data are means $\pm \mathrm{SD}$ or $n(\%)$, except for original values of triglycerides, alanine aminotransferase, and adiponectin levels, which are presented as medians (quartiles). $p$ values are between those with and without dysglycemia.

formed. Differences of continuous variables between two groups were evaluated by the unpaired $t$ test, whereas dichotomous variables between two groups were compared by the Fisher's exact test. A $p$ value for trend among more than two groups was assessed by the linear trend test in one-way ANOVA for continuous variables, and by the linear-by-linear association chi-squared test for dichotomous variables. In logistic regression analysis, continuous variables were not transformed into categorical variables but were entered as they were $[23,24]$. Odds ratios of the continuous variables were shown as those per one-SD increment, rather than those per one-unit increment [25], to make an easier inter-variable comparison in the association with dysglycemia. A $p$ value of less than 0.05 was considered to be significant and $95 \%$ confidence intervals (CI) were given when required. The statistical difference in the AUC was assessed through 10000time bootstrap resampling, with the use of $\mathrm{R}$ version 2.12.1 (R Development Core Team), whereas the other statistical analyses were performed using IBM SPSS Statistics Version 19 (SPSS Inc.).

\section{Results}

The characteristics of the study population are shown in Table 1. They were $50 \pm 9$ years old and 1015 (85\%) were male. The prevalence of dysglycemia detected by the 75 -g OGTT was $25 \%(n=296)$. Circulating adiponectin levels were significantly lower in the subjects with dysglycemia compared to those without it $(p<$ 0.01 ). The accumulation of metabolic risk factors was significantly associated with low circulating adiponectin levels and a high prevalence of dysglycemia (both $p$ for trend < 0.01) (Fig. 1).

Table 2 shows the association of characteristics with dysglycemia in the logistic regression models. Increased adiponectin levels had an inverse association with the risk of dysglycemia, independently of plasma glucose and hemoglobin A1c levels, as well as other covariates. The adjusted odds ratio and its $95 \% \mathrm{CI}$ of log-transformed adiponectin levels per one-SD increment were $0.7[0.6,0.8]$ in the $\mathrm{PG}+\mathrm{A} 1 \mathrm{c}+\mathrm{ADN}$ model, and $0.8[0.6,0.9]$ in the Full model (both $p<0.01$ ).

The ROC curves of these developed models for detecting dysglycemia are shown in Fig. 2A. The PG+A1c+ADN model had a significantly larger AUC than the PG+Alc model (0.746 vs. 0.729). The difference of the AUC and its 95\% CI were 0.017 $[0.004,0.031](p=0.01)$. However, the AUC of the $\mathrm{PG}+\mathrm{A} 1 \mathrm{c}+$ Other model was 0.815 and was much larger than that of the $\mathrm{PG}+\mathrm{A} 1 \mathrm{c}+\mathrm{ADN}$ model; the difference of AUC and its 95\% CI were 0.069 [0.039, 0.099] ( $p<$ 0.01 ). On the other hand, the AUC of the Full model 
A

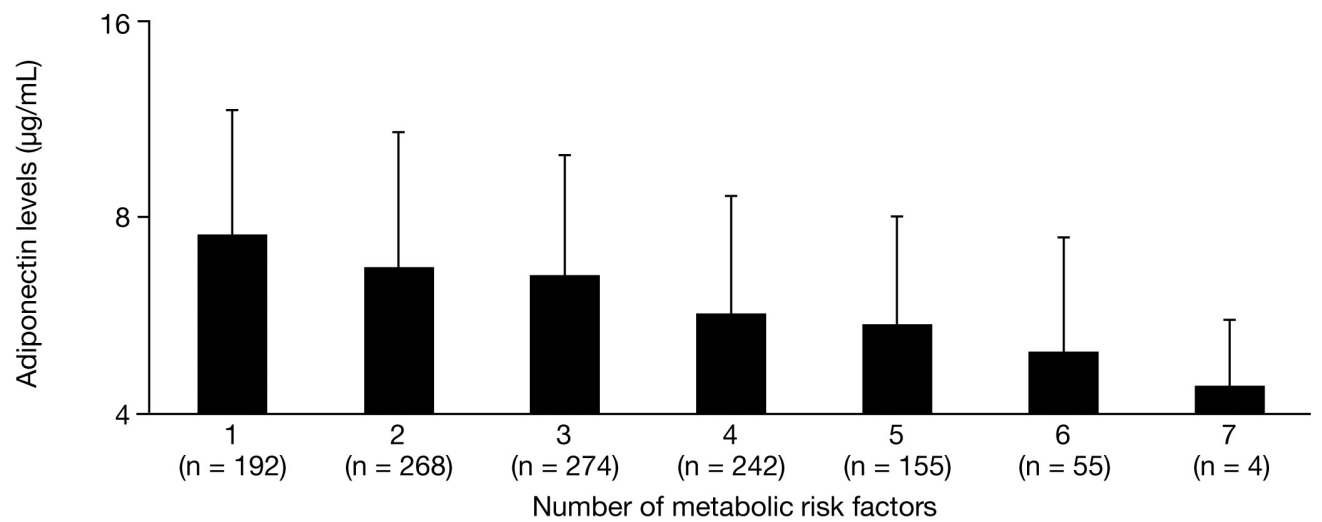

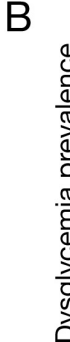

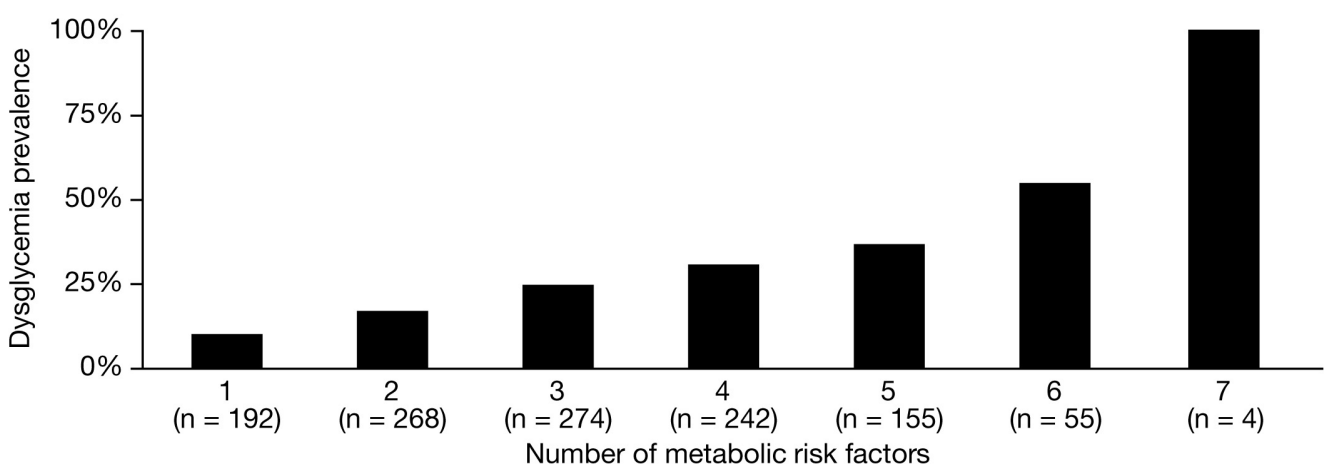

Fig. 1 Association of accumulating metabolic risk factors with circulating adiponectin levels (A) and the prevalence of dysglycemia (B) The study population was classified according to the number of accumulating metabolic risk factors. The metabolic risk factors were as follows (see Methods): (1) postprandial glucose levels $\geq 7.8 \mathrm{mmol} / \mathrm{L}$, (2) hemoglobin Alc levels $\geq 5.6 \%$, (3) elevated blood pressure, (4) abnormal lipid metabolism, (5) BMI $\geq 25 \mathrm{~kg} / \mathrm{m}^{2}$, (6) abdominal obesity, and/or (7) elevated serum alanine aminotransferase levels. Note that all individuals in the study population had one or more metabolic risk factors.

Table 2 Logistic regression models for dysglycemia

\begin{tabular}{|c|c|c|c|c|c|}
\hline & $\begin{array}{c}\text { Univariate } \\
\text { model }\end{array}$ & $\begin{array}{c}\mathrm{PG}+\mathrm{A} 1 \mathrm{c} \\
\text { model }\end{array}$ & $\begin{array}{c}\mathrm{PG}+\mathrm{A} 1 \mathrm{c}+\mathrm{ADN} \\
\text { model }\end{array}$ & $\begin{array}{c}\mathrm{PG}+\mathrm{A} 1 \mathrm{c}+\mathrm{Other} \\
\text { model }\end{array}$ & $\begin{array}{c}\text { Full } \\
\text { model }\end{array}$ \\
\hline Males & $1.1[0.8,1.7]$ & --- & --- & $\mathrm{N} / \mathrm{I}$ & $\mathrm{N} / \mathrm{I}$ \\
\hline Age & $2.0[1.7,2.3]^{*}$ & --- & --- & $1.8[1.5,2.2]^{*}$ & $1.9[1.6,2.4]^{*}$ \\
\hline Body mass index & $1.3[1.1,1.5]^{*}$ & --- & --- & $1.3[1.1,1.5]^{*}$ & $1.3[1.1,1.5]^{*}$ \\
\hline Systolic blood pressure & $1.5[1.3,1.7]^{*}$ & --- & --- & $1.3[1.1,1.5]^{*}$ & $1.3[1.1,1.5]^{*}$ \\
\hline Plasma glucose & $1.7[1.5,2.0]^{*}$ & $1.6[1.4,1.8]^{*}$ & $1.6[1.4,1.8]^{*}$ & $1.6[1.4,1.9]^{*}$ & $1.6[1.4,1.9]^{*}$ \\
\hline Hemoglobin A1c & $2.1[1.8,2.5]^{*}$ & $2.0[1.7,2.3]^{*}$ & $2.1[1.8,2.5]^{*}$ & $2.1[1.7,2.5]^{*}$ & $2.1[1.8,2.5]^{*}$ \\
\hline Total cholesterol & $1.1[1.0,1.3]$ & --- & --- & N/I & $\mathrm{N} / \mathrm{I}$ \\
\hline HDL cholesterol & $0.9[0.8,1.1]$ & --- & --- & N/I & $\mathrm{N} / \mathrm{I}$ \\
\hline Log (Triglycerides) & $1.3[1.1,1.4]^{*}$ & --- & --- & $\mathrm{N} / \mathrm{I}$ & $\mathrm{N} / \mathrm{I}$ \\
\hline Log (Alanine aminotransferase) & $1.5[1.3,1.7]^{*}$ & --- & --- & $1.8[1.5,2.2]^{*}$ & $1.7[1.4,2.0]^{*}$ \\
\hline Log (Adiponectin) & $0.8[0.7,0.9]^{*}$ & --- & $0.7[0.6,0.8]^{*}$ & --- & $0.8[0.6,0.9]^{*}$ \\
\hline Hypertension under treatment & $3.1[2.2,4.3]^{*}$ & --- & --- & $1.8[1.2,2.6]^{*}$ & $1.7[1.2,2.6]^{*}$ \\
\hline Dyslipidemia under treatment & $2.1[1.2,3.8]^{*}$ & --- & --- & $\mathrm{N} / \mathrm{I}$ & $\mathrm{N} / \mathrm{I}$ \\
\hline
\end{tabular}

Data are unadjusted odds ratios (OR) [95\% CI] in the Univariate model, and adjusted OR [95\% CI] in the other models. The OR [95\% $\mathrm{CI}$ ] of continuous variables are presented per one-SD increment. Asterisks show that the OR are statistically significant $(p<0.05)$. N/I, a candidate but not included in the final stepwise model. 
A
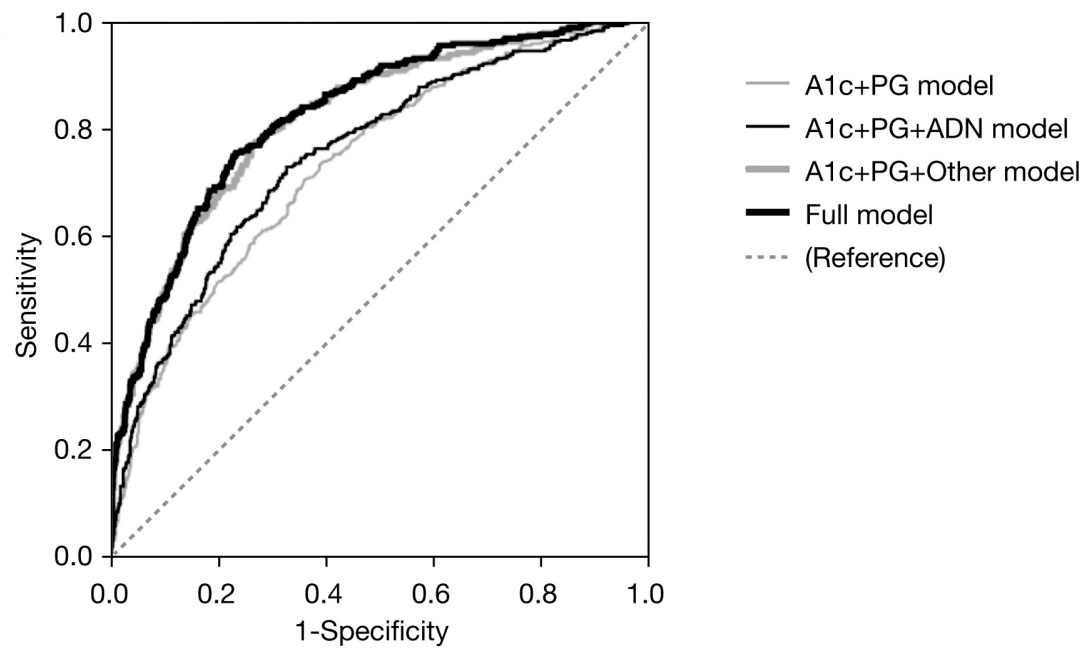

B
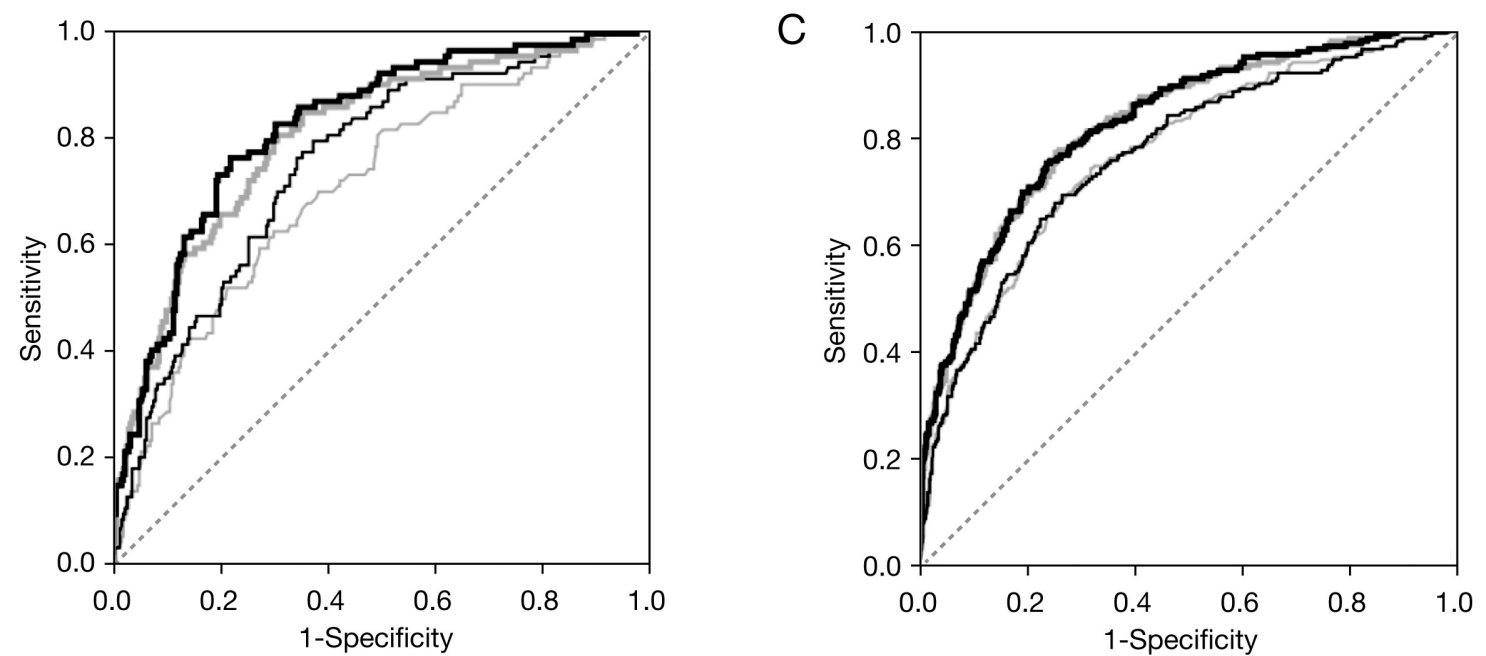

Fig. 2 Screening performance for detecting undiagnosed dysglycemia

ROC curves of the developed models are shown in the overall population (A), the subgroup without abdominal obesity (B), and those with abdominal obesity $(\mathbf{C})$. Thin gray line, the A1c+PG model; thin black line, the A1c+PG+ADN model; thick gray line, the A1c+PG+Other model; thick black line, the Full model. The AUC of the A1c+PG+ADN model (thin black line) was significantly larger than that of the A1c+PG model (thin gray line) in the overall population (A) and in the subgroup without abdominal obesity (B) (both $p=0.01$ ), but not in the subgroup with abdominal obesity $(\mathbf{C})(p=0.80)$. The Full model (thick black line) had a significantly larger AUC than the A1c+PG+Other model (thick gray line) in the subgroup without abdominal obesity (B) $(p=0.01)$, but not in the overall population $(\mathbf{A})(p=0.06)$ or in the subgroup with abdominal obesity $(\mathbf{C})(p=0.62)$.

was 0.821 , which was not significantly larger than that of the $\mathrm{PG}+\mathrm{A} 1 \mathrm{c}+\mathrm{Other}$ model; the difference and its $95 \%$ CI were $0.006[0.000,0.012](p=0.06)$.

We subsequently assessed the predictive capability in the subgroups with and without abdominal obesity. Fig. 2B and C show the ROC curves of the developed models for dysglycemia in the subgroups without and with abdominal obesity, respectively. In the subjects without abdominal obesity $(n=396)$, as shown in Fig. $2 \mathrm{~B}$, the $\mathrm{PG}+\mathrm{A} 1 \mathrm{c}+\mathrm{ADN}$ model had a significantly larger AUC than the PG+A1c model (0.747 vs. 0.705; $p=0.01)$. Furthermore, the AUC of the Full model was significantly larger than that of the $\mathrm{PG}+\mathrm{A} 1 \mathrm{c}+$ Other model (0.817 vs. 0.800; $p=0.01)$.

On the other hand, in the subjects with abdominal obesity $(n=794)$, the addition of adiponectin levels did not improve the predictive performance (Fig. 2C). The AUC of the A1c+PG+ADN model was 0.764 and was not significantly different from that of the $\mathrm{A} 1 \mathrm{c}+\mathrm{PG}$ model $(p=0.80, v s .0 .766)$. Neither was there any significant difference in the AUC between the Full model and the $\mathrm{A} 1 \mathrm{c}+\mathrm{PG}+$ Other model $(0.824 v s .0 .822 ; p=0.62)$. 


\section{Discussion}

The current study revealed that circulating adiponectin levels were independently associated with dysglycemia in non-fasting Japanese employees with metabolic risk factors, but that they improved the screening capacity only in those without abdominal obesity.

Early detection of dysglycemia is now regarded as an important strategy in healthcare fields [2], and health check-ups of employees has been widely performed at the workplace in Japan. However, there remains one challenging issue concerning health check-ups at the workplace; some employees have difficulty in undergoing health check-ups in the fasting state. In these subjects, the risk of dysglycemia is often assessed by postprandial plasma glucose levels, as well as hemoglobin A1c levels. As is often pointed out, the screening performance of postprandial examinations is inferior compared to fasting glucose levels [2]. A strategy to improve the screening performance in the non-fasting state is now highly desired in healthcare practice.

Circulating adiponectin level is one of the most promising biomarkers very close to practical use. Adiponectin is an adipocytokine constitutively produced by adipose tissue, whose circulating levels are inversely correlated with adiposity [26-28]. Recent studies demonstrated that its decreased levels are associated with various diseases, and dysglycemia is no exception [3-8]. However, most previous studies only investigated the relative risk of decreased adiponectin levels for dysglycemia, and did not assess its predictive performance, i.e, the area under the ROC curves (AUC). As recent studies demonstrated, a significant and independent association of a biomarker with an outcome, assessed with its relative risk, will not always guarantee its significant improvement of the predictive performance $[29,30]$. To assess its usefulness as a predictor, an evaluation of its predictive performance is needed [31]. Furthermore, it remains unknown whether the measurement of adiponectin levels is useful for the screening of dysglycemia in the non-fasting state. To the best of our knowledge, this is the first report concerning the improvement of screening performance by adiponectin levels in the non-fasting state.

In the current study, the PG+A1c+ADN model had a significantly larger AUC than the $\mathrm{PG}+\mathrm{A} 1 \mathrm{c}$ model in the overall population (Fig. 2A), indicating that the additional measurement of circulating adiponectin levels would significantly improve the screening performance compared to the combination of only postprandial glucose and hemoglobin A1c levels. However, the screening performance of the $\mathrm{PG}+\mathrm{A} 1 \mathrm{c}+\mathrm{ADN}$ model was significantly inferior compared to the $\mathrm{PG}+\mathrm{A} 1 \mathrm{c}+$ Other model (Fig. 1A). That is to say, the additional consideration of other routine health check-up data would be more informative than the additional measurement of adiponectin levels in the screening of dysglycemia. Furthermore, the AUC of the Full model (i.e., adding adiponectin levels to the $\mathrm{PG}+\mathrm{A} 1 \mathrm{c}+$ Other model) was not significantly different from that of the PG+A1c+Other model (Fig. 1A), although adiponectin levels were independently associated with dysglycemia (Table 2). These findings indicate that adiponectin levels would provide little additive information to other routine health check-up data in the screening of dysglycemia in the overall population. It is possible that adiponectin explains some effects of conventional risk factors on the development of dysglycemia, which would obscure the clinical usefulness of adiponectin measurements for predicting dysglycemia.

The current study also investigated whether the healthcare usefulness of adiponectin measurements would be consistent in subjects with and without abdominal obesity. Numerous previous studies have shown a strong inverse association between adiponectin and abdominal obesity [28]. In general, a ROC curve of a test value for an outcome could depend on the distribution of the test value [32]. Given that subjects with abdominal obesity are likely to have lower circulating adiponectin levels than those without it, adiponectin measurements might have different impacts on the predictive performance for dysglycemia between the two subgroups. Indeed, our study revealed that adding adiponectin measurements significantly improved the predictive performance in the subjects without abdominal obesity, but not in those with abdominal obesity (Fig. 2B and C). It is possible that in the subjects with abdominal obesity, coexisting metabolic abnormalities would already be so informative in predicting dysglycemia that adiponectin measurements would have little room for adding predictive information. Another possible explanation is that adiponectin levels might be low in most of the subjects with abdominal obesity [33]. Their relatively narrow distribution within a low range might cause their limited discriminative capability for dysglycemia prediction.

The current study had some limitations. First, the subjects in the current study had one or more metabolic 
risk factors. It still remains unknown whether the measurement of adiponectin levels would be useful in the subjects with no metabolic risk factors. Second, the current study did not assess high-molecular-weight adiponectin levels. They might show a different screening performance from that of total adiponectin levels. Third, the prevalence of male sex was high and relatively few females were included in the current study. Future studies with a sufficient number of females will be needed to validate the current findings.

In conclusion, circulating adiponectin levels were independently associated with dysglycemia in nonfasting Japanese employees with metabolic risk factors, but their measurements improved the screening capacity only in those without abdominal obesity.

\section{Acknowledgements}

Mitsuyoshi Takahara is a Research Fellow of the Japan Society for the Promotion of Science. There is no conflict of interest concerning this manuscript.

\section{References}

1. WHO Expert Committee on Health Promotion in the Work Setting (1988) Health Promotion for Working Populations. World Health Organization, Geneva.

2. Echouffo-Tcheugui JB, Ali MK, Griffin SJ, Narayan KM (2011) Screening for type 2 diabetes and dysglycemia. Epidemiol Rev 33: 63-87.

3. Li Y, Yatsuya H, Iso H, Toyoshima H, Tamakoshi K (2012) Inverse relationship of serum adiponectin concentration with type 2 diabetes mellitus incidence in middle-aged Japanese workers: six-year follow-up. Diabetes Metab Res Rev 28: 349-356.

4. Otsuka F, Sugiyama S, Kojima S, Maruyoshi H, Funahashi T, et al. (2007) Hypoadiponectinemia is associated with impaired glucose tolerance and coronary artery disease in non-diabetic men. Circ $J$ 71: 1703-1709.

5. Nakashima R, Kamei N, Yamane K, Nakanishi S, Nakashima A, et al. (2006) Decreased total and high molecular weight adiponectin are independent risk factors for the development of type 2 diabetes in JapaneseAmericans. J Clin Endocrinol Metab 91: 3873-3877.

6. Vendramini MF, Ferreira SR, Gimeno SG, Kasamatsu TS, Miranda WL, et al. (2006) Plasma adiponectin levels and incident glucose intolerance in JapaneseBrazilians: a seven-year follow-up study. Diabetes Res Clin Pract 73: 304-309.

7. Daimon M, Oizumi T, Saitoh T, Kameda W, Hirata A, et al. (2003) Decreased serum levels of adiponectin are a risk factor for the progression to type 2 diabetes in the Japanese Population: the Funagata study. Diabetes Care 26: 2015-2020.

8. LiS, Shin HJ, Ding EL, van Dam RM(2009)Adiponectin levels and risk of type 2 diabetes: a systematic review and meta-analysis. JAMA 302: 179-188.

9. Seino Y, Nanjo K, Tajima N, Kadowaki T, Kashiwagi A, et al. (2010) Report of the Committee on the classification and diagnostic criteria of diabetes mellitus: The Committee of the Japan Diabetes Society on the diag- nostic criteria of diabetes mellitus. $J$ Diabetes Invest 1 : 212-228

10. Miller M, Stone NJ, Ballantyne C, Bittner V, Criqui MH, et al. (2011) Triglycerides and cardiovascular disease: a scientific statement from the American Heart Association. Circulation 123: 2292-2333.

11. Ruckert IM, Heier M, Rathmann W, Baumeister SE, Doring A, et al. (2011) Association between markers of fatty liver disease and impaired glucose regulation in men and women from the general population: the KORA-F4-study. PLoS One 6: e22932.

12. Schindhelm RK, Diamant M, Dekker JM, Tushuizen ME, Teerlink T, et al. (2006) Alanine aminotransferase as a marker of non-alcoholic fatty liver disease in relation to type 2 diabetes mellitus and cardiovascular disease. Diabetes Metab Res Rev 22: 437-443.

13. Gariani K, Philippe J, Jornayvaz FR (2013) Nonalcoholic fatty liver disease and insulin resistance: from bench to bedside. Diabetes Metab 39: 16-26.

14. The Examination Committee of Criteria for the Metabolic Syndrome in Japan. (2005) Definition and diagnostic criteria of the metabolic syndrome. J Japan Soc Intern Med 94: 794-809 (in Japanese).

15. The Examination Committee of Criteria for 'Obesity Disease' in Japan and Japan Society for the Study of Obesity (2002) New criteria for 'obesity disease' in Japan. Circ J 66: 987-992.

16. Oka R, Yagi K, Sakurai M, Nakamura K, Nagasawa SY, et al. (2012) Impact of visceral adipose tissue and subcutaneous adipose tissue on insulin resistance in middle-aged Japanese. J Atheroscler Thromb 19: 814-822.

17. Takahara M, Kaneto H, Shimomura I (2013) Insulin Resistance can be Easily Estimated by Body Mass Index and Waist Circumference in a General Japanese Population. J Atheroscler Thromb 20: 401-403.

18. Bonora E, Kiechl S, Willeit J, Oberhollenzer F, Egger $\mathrm{G}$, et al. (1998) Prevalence of insulin resistance in metabolic disorders: the Bruneck Study. Diabetes 47: 1643- 
1649.

19. Alberti KG, Zimmet P, Shaw J (2006) Metabolic syndrome - a new world-wide definition. A Consensus Statement from the International Diabetes Federation. Diabet Med 23: 469-480.

20. Takahara M, Katakami N, Kaneto H, Noguchi M, Shimomura I (2013) Evaluation of Hypertriglyceridemia using Non-fasting Health Checkup Data in a Japanese Population. J Atheroscler Thromb 2013 Apr 22. [Epub ahead of print]

21. Nishimura A, Sawai T (2006) Determination of adiponectin in serum using a latex particle-enhanced turbidimetric immunoassay with an automated analyzer. Clin Chim Acta 371: 163-168.

22. Kashiwagi A, Kasuga M, Araki E, Oka Y, Hanafusa $\mathrm{T}$, et al. (2012) International clinical harmonization of glycated hemoglobin in Japan: From Japan Diabetes Society to National Glycohemoglobin Standardization Program values. J Diabetes Invest 3: 39-40.

23. Royston P, Altman DG, Sauerbrei W (2006) Dichotomizing continuous predictors in multiple regression: a bad idea. Stat Med 25: 127-141.

24. Fedorov V, Mannino F, Zhang R (2009) Consequences of dichotomization. Pharm Stat 8: 50-61.

25. May H (2004) Making statistics more meaningful for policy research and program evaluation. Am J Eval 25: 525-540.

26. Maeda K, Okubo K, Shimomura I, Funahashi T, Matsuzawa Y, et al. (1996) cDNA cloning and expression of a novel adipose specific collagen-like factor, apM1 (AdiPose Most abundant Gene transcript 1). Biochem Biophys Res Commun 221: 286-289.
27. Arita Y, Kihara S, Ouchi N, Takahashi M, Maeda K, et al. (1999) Paradoxical decrease of an adipose-specific protein, adiponectin, in obesity. Biochem Biophys Res Commun 257: 79-83.

28. Matsuzawa Y, Funahashi T, Nakamura T (2011) The concept of metabolic syndrome: contribution of visceral fat accumulation and its molecular mechanism. $J$ Atheroscler Thromb 18: 629-639.

29. Dallmeier D, Larson MG, Wang N, Fontes JD, Benjamin EJ, et al. (2012) Addition of inflammatory biomarkers did not improve diabetes prediction in the community: the framingham heart study. J Am Heart Assoc 1: e000869.

30. Wilson PW, Nam BH, Pencina M, D'Agostino RB, Sr., Benjamin EJ, et al. (2005) C-reactive protein and risk of cardiovascular disease in men and women from the Framingham Heart Study. Arch Intern Med 165: 24732478.

31. Sattar N, Wannamethee SG, Forouhi NG (2008) Novel biochemical risk factors for type 2 diabetes: pathogenic insights or prediction possibilities? Diabetologia 51: 926-940.

32. Takahara M, Katakami N, Kaneto H, Noguchi M, Shimomura I (2012) Statistical reassessment of the association between waist circumference and clustering metabolic abnormalities in Japanese population. $J$ Atheroscler Thromb 19: 767-778.

33. Saito T, Murata M, Otani T, Tamemoto H, Kawakami M, et al. (2012) Association of subcutaneous and visceral fat mass with serum concentrations of adipokines in subjects with type 2 diabetes mellitus. Endocr J 59: $39-45$. 